\title{
Linear vibrations of continuum with fractional derivatives
}

\author{
Duygu Dönmez Demir ${ }^{{ }^{*}}$, Necdet Bildik ${ }^{1}$ and Berra Gültekin Sinir ${ }^{2}$
}

"Correspondence:

duygu.donmez@cbu.edu.tr

'Department of Mathematics,

Faculty of Art \& Sciences, Celal Bayar

University, Manisa, 45047, Turkey

Full list of author information is

available at the end of the article

\begin{abstract}
In this paper, linear vibrations of axially moving systems which are modelled by a fractional derivative are considered. The approximate analytical solution is obtained by applying the method of multiple scales. Including stability analysis, the effects of variation in different parameters belonging to the application problems on the system are calculated numerically and depicted by graphs. It is determined that the external excitation force acting on the system has an effect on the stiffness of the system. Moreover, the general algorithm developed can be applied to many problems for linear vibrations of continuum.
\end{abstract}

Keywords: linear vibrations; dynamic analysis of continuum; fractional derivative; perturbation method

\section{Introduction}

Fractional derivatives are useful for describing the occurrence of vibrations in engineering practice. The studies involving fractional calculus and its applications to mechanical problems appear widely in different studies [1]. The advances in fractional calculus focus on modern examples in differential and integral equations, physics, signal processing, fluid mechanics, viscoelasticity, mathematical biology and electrochemistry [2].

The general solution procedure including all the problems instead of separately solving each problem is quite advantageous. Many different linear or nonlinear models addressing vibrations of continuum appear in the literature. Some of these works are as follows: Pakdemirli [3] developed a general operator technique to analyse the vibrations of a continuous system with an arbitrary number of coupled differential equations. Özhan and Pakdemirli [4-6] suggested the general solution procedure to investigate a more general class of continuous systems such as gyroscopic and viscoelastic systems. Ghayesh et al. [7] considered a general solution procedure for the vibrations of systems with cubic nonlinearities subjected to nonlinear and time-dependent boundary conditions. Hence, a general solution is adapted to solve the dynamic problems constituting continuum.

In recent years, there has been a growing interest in the area of fractional variational calculus and its applications [8, 9]. Fractional calculus, which is used successfully in various fields such as mathematics, science and engineering, is one of the generalisations of classical calculus. The merits of using a fractional differential operator lie in the fact that few parameters are needed to accurately describe the constitutive law of damping ma-

\section{黛 Springer}

C) 2013 Dönmez Demir et al: licensee Springer. This is an Open Access article distributed under the terms of the Creative Commons Attribution License (http://creativecommons.org/licenses/by/2.0), which permits unrestricted use, distribution, and reproduction in any medium, provided the original work is properly cited. 
terials [10]. Bagley and Calico [11] modelled the mechanical properties of damping materials by fractional order time derivatives. The mechanical scientific community recognised the significance of fractional calculus for modelling viscoelastic material behaviour thanks to Bagley et al. [12]. Also, they studied longitudinal vibrations of rods and flexural vibrations of beams based on viscoelastic fractional derivative models [13]. For solving dynamic problems with a fractional derivative, the analysis of free damped vibrations of various mechanical systems, whose behaviour is described by linear viscoelastic models with fractional derivatives, were studied by Rossikhin and Shitikova [14]. Mainardi [15] considered the problems in continuum mechanics related to mathematical modelling of viscoelastic bodies. Cooke et al. [16] investigated the response of a viscoelastic beam with a fractional derivative. Skaar et al. [17] used a fractional standard linear solid model. French and Rogers [18] presented a small group of structural dynamics problems for which fractional calculus was adopted.

The general solution allows one to investigate the effects on a dynamic analysis of continuum whose damping term is modelled by a fractional derivative. An engineering problem which is a special application of the general model developed in this study was formerly considered in [19]. In our previous study [20], the analysis of primary and parametric resonance for the external excitation term having $\varepsilon$-order was performed. As the forced term is obtained in one-order, sum or difference type of resonance also appears in the present model. The method of multiple scales is used in the analysis. Thus, the amplitude and phase modulation equations are produced in terms of operators. In addition, the variations of the curves with respect to the dimensionless parameters are presented. Finally, the effects of fractional damping on the linear vibrations of continuum are investigated in detail.

\section{Equation of motion}

Let us consider a non-homogeneous and dimensionless model as follows:

$$
\begin{aligned}
& \ddot{w}+L_{0}[w]+\varepsilon\left\{L_{1}[w] \cos \Omega_{1} t+L_{2}\left[D^{\alpha} w\right]\right\}=F(x) \cos \Omega_{2} t, \\
& B_{1}(w)=0 \quad \text { at } x=0, \quad B_{2}(w)=0 \quad \text { at } x=1,
\end{aligned}
$$

where $w(x, t)$ represents the displacement, $x$ and $t$ are the spatial and time variables. $\varepsilon$ is a small dimensionless parameter, $F$ is the external excitation force, $\Omega_{1}$ and $\Omega_{2}$ are the internal and the external excitation frequencies, respectively. $D^{\alpha}$ defines the fractional derivative of order $\alpha$. The dot denotes differentiation with respect to time $t ; L_{0}, L_{1}$ and $L_{2}$ are self-adjoint operators involving only the spatial variable $x, B_{1}$ and $B_{2}$ are linear operators of boundary conditions. Here, the associated boundary conditions are linear, homogeneous and free from the time.

\section{Method of multiple scales}

The method is directly applied to the partial differential equation (1). Thus, we can write

$$
w(x, t ; \varepsilon)=w_{0}\left(x, T_{0}, T_{1}\right)+\varepsilon w_{1}\left(x, T_{0}, T_{1}\right)+\cdots,
$$


where $t=T_{0}$ is the usual fast-time scales, $\varepsilon t=T_{1}$ is the slow-time scales. Time derivatives are expressed in terms of fast and slow time scales as follows [21]:

$$
d / d t=D_{0}+\varepsilon D_{1}+\cdots, \quad d^{2} / d t^{2}=D_{0}^{2}+2 \varepsilon D_{0} D_{1}+\cdots,
$$

where $D_{n}=\partial / \partial T_{n}$ and $T_{n}=\varepsilon^{n} t$. The perturbative expansion of the Riemann-Liouville fractional derivative is given by

$$
\left(\frac{d}{d t}\right)^{\alpha}=D_{+}^{\alpha}+\varepsilon \alpha D_{+}^{\alpha-1} D_{1}+\frac{1}{2} \varepsilon^{2} \alpha\left[(\alpha-1) D_{+}^{\alpha-2} D_{1}^{2}+2 D_{+}^{\alpha-1} D_{2}\right]+\cdots
$$

In order to calculate the fractional derivative of the exponential function, we may use Riemann-Liouville derivatives [22]

$$
D_{+}^{\alpha} u(t)=\frac{1}{\Gamma(1-\alpha)} \frac{d}{d t} \int_{-\infty}^{t} \frac{u(\tau) d \tau}{(t-\tau)^{\alpha}} .
$$

If we take $u(t)=e^{i \omega t}$, then

$$
D_{+}^{\alpha} e^{i \omega t}=(i \omega)^{\alpha} e^{i \omega t}
$$

is obtained such that $D_{+}^{\alpha}, D_{+}^{\alpha-1}, D_{+}^{\alpha-2}, \ldots$ are the Riemann-Liouville fractional derivatives. Substituting Eqs. (3)-(5) into Eqs. (1) and (2), one obtains

$$
\begin{aligned}
& O(1): D_{0}^{2} w_{0}+L_{0}\left[w_{0}\right]=F(x) \cos \Omega_{2} T_{0}, \\
& B_{1}\left(w_{0}\right)=0 \quad \text { at } x=0, \quad B_{2}\left(w_{0}\right)=0 \quad \text { at } x=1, \\
& O(\varepsilon): D_{0}^{2} w_{1}+L_{0}\left[w_{1}\right]=-2 D_{0} D_{1} w_{0}-L_{1}\left[w_{0}\right] \cos \Omega_{1} T_{0}-L_{2}\left[D_{0} w_{0}\right], \\
& B_{1}\left(w_{1}\right)=0 \quad \text { at } x=0, \quad B_{2}\left(w_{1}\right)=0 \quad \text { at } x=1 .
\end{aligned}
$$

The solution at $\varepsilon^{0}$-order is

$$
w_{0}\left(x, T_{0}, T_{1}\right)=\left[A_{n}\left(T_{1}\right) e^{i \omega_{n} T_{0}}+c c\right] X_{n}(x)+\left(e^{i \Omega_{2} T_{0}}+c c\right) Y(x),
$$

where $c c$ denotes complex conjugates. On the other hand, the functions $X_{n}$ and $Y$ satisfy the following equations:

$$
\begin{aligned}
& L_{0}\left[X_{n}\right]-\omega_{n}^{2} X_{n}=0 ; \quad n=1,2, \ldots, \\
& B_{1}\left(X_{n}\right)=0 \quad \text { at } x=0 \quad \text { and } \quad B_{2}\left(X_{n}\right)=0 \quad \text { at } x=1, \\
& L_{0}[Y]-\Omega_{2}^{2} Y=\frac{1}{2} F, \\
& B_{1}(Y)=0 \quad \text { at } x=0, \quad B_{2}(Y)=0 \quad \text { at } x=1 .
\end{aligned}
$$

At $\varepsilon$-order, the solution is

$$
w_{1}\left(x, T_{0}, T_{1}\right)=\phi_{n}\left(x, T_{1}\right) e^{i \omega_{n} T_{0}}+c c+W\left(x, T_{0}, T_{1}\right),
$$


where $W\left(x, T_{0}, T_{1}\right)$ is related to the non-secular terms and other parts of the solution are associated with the secular terms. For the approximate solution at $\varepsilon$-order, one substitutes Eq. (12) into Eq. (10). Thus, the resulting equation is obtained as

$$
\begin{aligned}
D_{0}^{2} w_{1}+L_{0}\left[w_{1}\right]= & \left.-2 i \omega_{n} D_{1} A_{n} e^{i \omega_{n} T_{0}}+2 i \omega_{n} D_{1} \bar{A}_{n} e^{-i \omega_{n} T_{0}}\right] X_{n}(x) \\
& -\frac{1}{2} L_{1}\left[X_{n}\right]\left[A_{n}\left(e^{i\left(\omega_{n}+\Omega_{1}\right) T_{0}}+e^{i\left(\omega_{n}-\Omega_{1}\right) T_{0}}\right)+c c\right] \\
& -\frac{1}{2} L_{1}[Y]\left[e^{i\left(\Omega_{1}+\Omega_{2}\right) T_{0}}+e^{i\left(\Omega_{1}-\Omega_{2}\right) T_{0}}+c c\right] \\
& -L_{2}\left[X_{n}\right]\left[\left(i \omega_{n}\right)^{\alpha} A_{n} e^{i \omega_{n} T_{0}}+\left(-i \omega_{n}\right)^{\alpha} \bar{A}_{n} e^{-i \omega_{n} T_{0}}\right] \\
& -L_{2}[Y]\left[\left(i \Omega_{2}\right)^{\alpha} e^{i \Omega_{2} T_{0}}+\left(-i \Omega_{2}\right)^{\alpha} e^{-i \Omega_{2} T_{0}}\right] .
\end{aligned}
$$

Then, five cases occur as follows.

\section{Case studies}

In this section, we assume that one dominant mode of vibrations exists. Depending on the numerical values of natural frequency, five different cases occur.

\section{1 $\Omega_{1}$ away from $2 \omega_{n}$ and $0, \Omega_{2}$ away from $\omega_{n}$}

This case corresponds to absence of any resonances. Then Eq. (18) turns into

$$
D_{0}^{2} w_{1}+L_{0}\left[w_{1}\right]=\left[-2 i \omega_{n} D_{1} A_{n} X_{n}-\left(i \omega_{n}\right)^{\alpha} A_{n} L_{2}\left[X_{n}\right]\right] e^{i \omega_{n} T_{0}}+c c+N S T,
$$

where NST denotes non-secular terms. If Eq. (17) is substituted into Eq. (19), then $\phi_{n}$ satisfies

$$
\begin{aligned}
& L_{0}\left[\phi_{n}\right]-\omega_{n}^{2} \phi_{n}=-2 i \omega_{n} D_{1} A_{n} X_{n}-\left(i \omega_{n}\right)^{\alpha} A_{n} L_{2}\left[X_{n}\right], \\
& B_{1}\left(\phi_{n}\right)=0 \quad \text { at } x=0, \quad B_{2}\left(\phi_{n}\right)=0 \quad \text { at } x=1 .
\end{aligned}
$$

Thus, the solvability condition [23] requires

$$
2 i \omega_{n} D_{1} A_{n}+\left(i \omega_{n}\right)^{\alpha} d_{1} A_{n}=0,
$$

where

$$
d_{1}=\int_{0}^{1} X_{n}(x) L_{2}\left[X_{n}\right] d x
$$

Finally, the amplitude is obtained as

$$
A_{n}\left(T_{1}\right)=A_{0} \exp \left(\frac{1}{2} \omega_{n}^{\alpha-1} d_{1}\left[i \cos \left(\frac{\pi}{2} \alpha\right)-\sin \left(\frac{\pi}{2} \alpha\right)\right] T_{1}\right)
$$


and in the same sense, the displacement is calculated as

$$
\begin{aligned}
w(x, t) \cong & A_{0}\left\{\exp \left(-\frac{d_{1}}{2} \omega_{n}^{\alpha-1} \sin \left(\frac{\pi}{2} \alpha\right) \varepsilon t\right)\right. \\
& \left.\times\left[\exp \left[i\left(\frac{d_{1}}{2} \varepsilon \omega_{n}^{\alpha-1} \cos \left(\frac{\pi}{2} \alpha\right)+\omega_{n}\right) t\right]+c c\right]\right\} X_{n}(x) \\
& +\left(e^{i \Omega_{2} t}+e^{-i \Omega_{2} t}\right) Y(x),
\end{aligned}
$$

where $A_{0}$ is constant.

\section{2 $\Omega_{1}$ close to $2 \omega_{n}$ and $\Omega_{2}$ away from $\omega_{n}$}

Principal parametric resonance occurs in this case. Thus, the internal excitation frequency is considered as

$$
\Omega_{1}=2 \omega_{n}+\varepsilon \sigma_{n},
$$

where $\sigma_{n}$ is a detuning parameter. Then Eq. (18) becomes

$$
2 i \omega_{n} D_{1} A_{n}+\frac{1}{2} d_{2} \bar{A}_{n} e^{i \sigma_{n} T_{1}}+\left(i \omega_{n}\right)^{\alpha} d_{1} A_{n}=0
$$

where

$$
d_{2}=\int_{0}^{1} X_{n}(x) L_{1}\left[X_{n}\right] d x
$$

For the stability analysis, one introduces the transformation

$$
A_{n}\left(T_{1}\right)=B_{n}\left(T_{1}\right) e^{i \sigma_{n} T_{1} / 2},
$$

where

$$
B_{n}\left(T_{1}\right)=\left(b_{n}^{R}+i b_{n}^{I}\right) e^{\lambda T_{1}} .
$$

Substituting (29) and (30) into Eq. (27), and separating real and imaginary parts, the representation of the system of equations with the coefficient matrix is given as

$$
\left[\begin{array}{cc}
\lambda+\frac{d_{1}}{2} \omega_{n}^{\alpha-1} \sin \left(\frac{\pi}{2} \alpha\right) & -\frac{d_{2}}{4 \omega_{n}}+\left(\frac{d_{1}}{2} \omega_{n}^{\alpha-1} \cos \left(\frac{\pi}{2} \alpha\right)-\frac{\sigma_{n}}{2}\right) \\
-\frac{d_{2}}{4 \omega_{n}}-\left(\frac{d_{1}}{2} \omega_{n}^{\alpha-1} \cos \left(\frac{\pi}{2} \alpha\right)-\frac{\sigma_{n}}{2}\right) & \lambda+\frac{d_{1}}{2} \omega_{n}^{\alpha-1} \sin \left(\frac{\pi}{2} \alpha\right)
\end{array}\right]\left[\begin{array}{l}
b_{n}^{R} \\
b_{n}^{I}
\end{array}\right]=\left[\begin{array}{l}
0 \\
0
\end{array}\right] .
$$

For a non-trivial solution $\left(b_{n}^{R} \neq 0, b_{n}^{I} \neq 0\right)$, the determinant of the coefficient matrix must be

$$
\left[\lambda+\frac{d_{1}}{2} \omega_{n}^{\alpha-1} \sin \left(\frac{\pi}{2} \alpha\right)\right]^{2}-\left[\left(\frac{d_{2}}{4 \omega_{n}}\right)^{2}-\left(\frac{d_{1}}{2} \omega_{n}^{\alpha-1} \cos \left(\frac{\pi}{2} \alpha\right)-\frac{\sigma_{n}}{2}\right)^{2}\right]=0
$$

For the steady state condition, $\lambda$ must be zero. Therefore, the stability boundaries are

$$
\sigma_{n}=d_{1} \omega_{n}^{\alpha-1} \cos \left(\frac{\pi}{2} \alpha\right) \pm \sqrt{\frac{d_{2}^{2}}{4 \omega_{n}^{2}}-d_{1}^{2} \omega_{n}^{2 \alpha-2} \sin ^{2}\left(\frac{\pi}{2} \alpha\right)} .
$$


Inserting $\sigma_{n}$ into Eq. (26), the internal excitation frequency is obtained as

$$
\Omega_{1} \cong 2 \omega_{n}+\varepsilon\left[d_{1} \omega_{n}^{\alpha-1} \cos \left(\frac{\pi}{2} \alpha\right) \pm \sqrt{\left(\frac{d_{2}}{2 \omega_{n}}\right)^{2}-\left(d_{1} \omega_{n}^{\alpha-1} \sin \left(\frac{\pi}{2} \alpha\right)\right)^{2}}\right]
$$

\section{3 $\Omega_{1}$ close to 0 and $\Omega_{2}$ away from $\omega_{n}$}

The nearness of $\Omega_{1}$ to zero is expressed as

$$
\Omega_{1}=\varepsilon \sigma_{n} .
$$

Arranging Eq. (18), one obtains

$$
2 i \omega_{n} D_{1} A_{n}+\left[\cos \left(\sigma_{n} T_{1}\right) d_{2}+\left(i \omega_{n}\right)^{\alpha} d_{1}\right] A_{n}=0
$$

Solving Eq. (36),

$$
\begin{aligned}
A_{n}\left(T_{1}\right)= & A_{0} \exp \left[-\frac{d_{1}}{2} \omega_{n}^{\alpha-1} \sin \left(\frac{\pi}{2} \alpha\right) T_{1}\right. \\
& \left.+\frac{i}{2}\left(\frac{d_{2}}{\omega_{n} \sigma_{n}} \sin \left(\sigma_{n} T_{1}\right)+d_{1} \omega_{n}^{\alpha-1} \cos \left(\frac{\pi}{2} \alpha\right) T_{1}\right)\right]
\end{aligned}
$$

and

$$
\begin{aligned}
w(x, t) \cong & A_{0}\left\{\exp \left(-\frac{d_{1}}{2} \omega_{n}^{\alpha-1} \sin \left(\frac{\pi}{2} \alpha\right) \varepsilon t\right)\right. \\
& \left.\times\left[\exp \left(\frac{i}{2}\left[\frac{d_{2}}{\omega_{n} \sigma_{n}} \sin \left(\sigma_{n} \varepsilon t\right)+\varepsilon d_{1} t \omega_{n}^{\alpha-1} \cos \left(\frac{\pi}{2} \alpha\right)+\omega_{n} t\right]\right)+c c\right]\right\} X_{n}(x) \\
& +\left(e^{i \Omega_{2} T_{0}}+e^{-i \Omega_{2} T_{0}}\right) Y(x)
\end{aligned}
$$

is calculated.

\section{4 $\Omega_{1}$ away from $2 \omega_{n}$ and $\Omega_{2}$ close to $\omega_{n}$}

In this case, we consider the primary resonance $\Omega_{2} \cong \omega_{n}$ when the frequency of the loading is approximately equal to the natural frequency. Thus, Eq. (18) turns into

$$
2 i \omega_{n} D_{1} A_{n}+\left(i \omega_{n}\right)^{\alpha} d_{1} A_{n}+\left(i \omega_{n}\right)^{\alpha} d_{3} e^{i \sigma_{n} T_{1}}=0
$$

where

$$
d_{3}=\int_{0}^{1} X_{n} L_{2}[Y] d x
$$

Substituting the polar form

$$
A_{n}\left(T_{1}\right)=\frac{1}{2} a_{n}\left(T_{1}\right) e^{i \beta_{n}\left(T_{1}\right)}
$$


into Eq. (39) and separating the equation into real and imaginary parts, we obtain

$$
\begin{gathered}
\operatorname{Re}: a_{n}^{\prime}=-\frac{d_{1}}{2} \omega_{n}^{\alpha-1} a_{n} \sin \left(\frac{\pi}{2} \alpha\right)-d_{3} \omega_{n}^{\alpha-1}\left[\cos \left(\frac{\pi}{2} \alpha\right) \sin \gamma_{n}+\sin \left(\frac{\pi}{2} \alpha\right) \cos \gamma_{n}\right], \\
\operatorname{Im}: \sigma_{n}-\gamma_{n}^{\prime}=\frac{d_{1}}{2} \omega_{n}^{\alpha-1} \cos \left(\frac{\pi}{2} \alpha\right) \\
+\frac{d_{3}}{a_{n}} \omega_{n}^{\alpha-1}\left(\cos \left(\frac{\pi}{2} \alpha\right) \cos \gamma_{n}-\sin \left(\frac{\pi}{2} \alpha\right) \sin \gamma_{n}\right),
\end{gathered}
$$

where $\gamma_{n}=\sigma_{n} T_{1}-\beta_{n}$. For steady-state solutions, we consider

$$
a_{n}^{\prime}=\gamma_{n}^{\prime}=0
$$

By the same mathematical manipulation, the stability boundaries are found as

$$
\sigma_{n}=\frac{d_{1}}{2} \omega_{n}^{\alpha-1} \cos \left(\frac{\pi}{2} \alpha\right) \pm \sqrt{\frac{d_{3}^{2} \omega_{n}^{2 \alpha-2}}{a_{n}^{2}}-\frac{d_{1}^{2}}{4} \omega_{n}^{2 \alpha-2} \sin ^{2}\left(\frac{\pi}{2} \alpha\right)}
$$

\subsection{Sum and difference type of resonance}

Let us consider sum or difference of internal and external excitation frequency as $\Omega_{1} \neq 0$, $\Omega_{1} \neq 2 \omega_{n}$ and $\Omega_{2} \neq \omega_{n}$. Then $\Omega_{1}+\Omega_{2}$ and $\Omega_{1}-\Omega_{2}, \Omega_{2}-\Omega_{1}$ are close to $\omega_{n}$. If Eq. (18) is arranged, we get

$$
2 i \omega_{n} D_{1} A_{n}+\frac{1}{2} d_{4} e^{i \sigma_{n} T_{1}}+\left(i \omega_{n}\right)^{\alpha} d_{1} A_{n}=0
$$

where

$$
d_{4}=\int_{0}^{1} X_{n} L_{1}[Y] d x
$$

Substituting Eq. (41) into Eq. (46) and separating into real and imaginary parts, we get

$$
\begin{aligned}
& \operatorname{Re}: a_{n}^{\prime}+\frac{d_{1}}{2} \omega_{n}^{\alpha-1} a_{n} \sin \left(\frac{\pi}{2} \alpha\right)=-\frac{d_{4}}{2 \omega_{n}} \sin \gamma_{n}, \\
& \operatorname{Im}: \sigma_{n}-\gamma_{n}^{\prime}-\frac{d_{1}}{2} \omega_{n}^{\alpha-1} \cos \left(\frac{\pi}{2} \alpha\right)=\frac{d_{4}}{2 \omega_{n} a_{n}} \cos \gamma_{n} .
\end{aligned}
$$

For steady-state solutions, the equations must be rearranged according to the condition (44). Then the stability boundaries are obtained as

$$
\sigma_{n}=\frac{d_{1}}{2} \omega_{n}^{\alpha-1} \cos \left(\frac{\pi}{2} \alpha\right) \pm \frac{1}{2} \sqrt{\frac{d_{4}^{2}}{\omega_{n}^{2} a_{n}^{2}}-d_{1}^{2} \omega_{n}^{2 \alpha-2} \sin ^{2}\left(\frac{\pi}{2} \alpha\right)} .
$$

\section{Applications}

\subsection{The longitudinal vibrations of a tensioned rod}

We will investigate longitudinal vibrations of an axial loaded rod with linear fractional damping for application. This problem is quite important in engineering applications. Also, the rods are used as a structural element in many civil and mechanical engineer- 
ing problems. The governing equation motion of a tensioned rod with fractional damping is introduced as

$$
m \frac{\partial^{2} \hat{w}}{\partial \hat{t}^{2}}-P \frac{\partial^{2} \hat{w}}{\partial \hat{x}^{2}}+\hat{\eta} \frac{\partial^{\alpha} \hat{w}}{\partial \hat{t}^{\alpha}}=0
$$

where $\hat{w}(\hat{x}, \hat{t})$ is the longitudinal displacement of the rod, $\varepsilon$ is a small dimensionless parameter, $m$ denotes the mass and $\hat{\eta}$ defines the damping coefficient. It is assumed that the tension $P$ is characterised as a small periodic perturbation $\varepsilon P_{1} \cos \hat{\Omega} \hat{t}$ on the steady-state tension $P_{0}$

$$
P=P_{0}+\varepsilon P_{1} \cos \hat{\Omega} \hat{t}
$$

where $\hat{\Omega}$ is the frequency of the rod [24]. Introducing the dimensionless quantities as

$$
w=\frac{\hat{w}}{L}, \quad x=\frac{\hat{x}}{L}, \quad t=\frac{\hat{t}}{L} \sqrt{\frac{P_{0}}{m}},
$$

the new dimensionless parameters are

$$
N=\frac{P_{1}}{P_{0}}, \quad \bar{\eta}=\frac{\hat{\eta}}{L^{\alpha-2}} \sqrt{\frac{P_{0}^{\alpha-2}}{m^{\alpha}}} \quad(\bar{\eta}=\varepsilon \eta), \quad \Omega=\hat{\Omega} L \sqrt{\frac{m}{P_{0}}},
$$

where $L$ is the length of the rod. Thus, the dimensionless equation is presented as

$$
\frac{\partial^{2} w}{\partial t^{2}}-(1+\varepsilon N \cos \Omega t) \frac{\partial^{2} w}{\partial x^{2}}+\varepsilon \eta \frac{\partial^{\alpha} w}{\partial t^{\alpha}}=0
$$

For the simply supported beam, the boundary conditions are

$$
w(0, t)=w(1, t)=0
$$

Considering Eq. (55), the operators corresponding to the general model are

$$
\begin{aligned}
& L_{0}[w]=-w^{\prime \prime}, \\
& L_{1}[w]=-N w^{\prime \prime}, \\
& L_{2}[w]=\eta D^{\alpha} w
\end{aligned}
$$

and the other terms are

$$
\begin{aligned}
& F(x)=0, \\
& \Omega_{1}=\Omega .
\end{aligned}
$$

Thus, the spatial function $X_{n}(x)$ satisfies

$$
\begin{aligned}
& X_{n}^{\prime \prime}+\omega_{n}^{2} X_{n}=0, \\
& X_{n}(0)=X_{n}(1)=0 .
\end{aligned}
$$


Finally, the solution of eigenvalue-eigenfunction problem (62) is

$$
X_{n}(x)=c_{2 n} \sin \omega_{n} x ; \quad \omega_{n}=n \pi, n=1,2, \ldots
$$

In this problem, three different cases arise at $\varepsilon$-order as follows.

5.1.1 $\Omega_{1}$ away from $2 \omega_{n}$ and $0, \Omega_{2}$ away from $\omega_{n}$ By the general solution (23), we may write

$$
d_{1}=\eta \int_{0}^{1} X_{n}^{2}(x) d x
$$

Thus, the displacement is obtained as

$$
\begin{aligned}
w(x, t) \cong & A_{0} \exp \left(-\frac{\eta}{2} \omega_{n}^{\alpha-1} \varepsilon t \sin \left(\frac{\pi}{2} \alpha\right)\right) \\
& \times\left[\exp \left(i \frac{\eta}{2} \varepsilon t \omega_{n}^{\alpha-1} \cos \left(\frac{\pi}{2} \alpha\right)+i \omega_{n} t\right)+c c\right] \sin \omega_{n} x .
\end{aligned}
$$

In Figure 1, it is observed that the damping and the natural frequency changed for different modes. The damping decreases and the natural frequency increases as the number of modes enlarges.

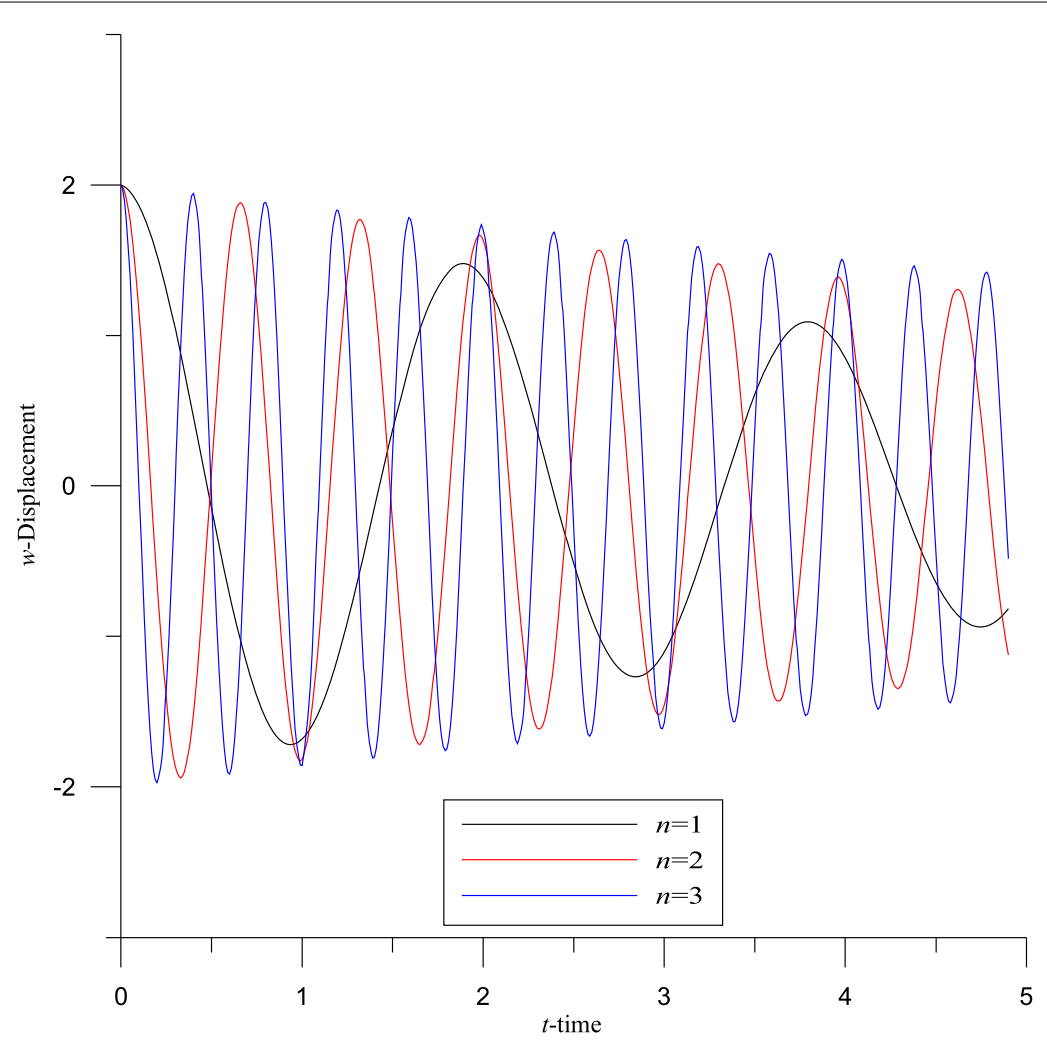

Figure 1 Displacement-time graph for different mode values $(\varepsilon=0.1, \eta=8, \alpha=0.5)$. 
5.1.2 $\Omega_{1}$ close to 0 and $\Omega_{2}$ away from $\omega_{n}$

This case corresponds to $\Omega_{1}=\varepsilon \sigma_{n}$. Using Eq. (28), we get

$$
d_{2}=-N \int_{0}^{1} X_{n}(x) X_{n}^{\prime \prime}(x) d x
$$

Thus, the amplitude and displacement are calculated respectively as follows:

$$
\begin{aligned}
A_{n}\left(T_{1}\right)= & A_{0} \exp \left\{-\frac{\eta}{2} \omega_{n}^{\alpha-1} T_{1} \sin \left(\frac{\pi}{2} \alpha\right)+i\left[-\frac{N}{2 \omega_{n} \sigma_{n}} \sin \left(\sigma_{n} T_{1}\right) \int_{0}^{1} X_{n}(x) X_{n}^{\prime \prime}(x) d x\right.\right. \\
& \left.\left.+\frac{\eta}{2} \omega_{n}^{\alpha-1} T_{1} \cos \left(\frac{\pi}{2} \alpha\right)\right]\right\}
\end{aligned}
$$

and

$$
\begin{aligned}
w(x, t) \cong & A_{0}\left\{\operatorname { e x p } ( - \frac { \eta } { 2 } \omega _ { n } ^ { \alpha - 1 } \varepsilon t \operatorname { s i n } ( \frac { \pi } { 2 } \alpha ) ) \left[\operatorname { e x p } \left(-i \frac{N}{2 \omega_{n} \sigma_{n}} \sin \left(\sigma_{n} \varepsilon t\right) \int_{0}^{1} X_{n} X_{n}^{\prime \prime} d x\right.\right.\right. \\
& \left.\left.\left.+i \frac{\eta}{2} \varepsilon t \omega_{n}^{\alpha-1} \cos \left(\frac{\pi}{2} \alpha\right)+i \omega_{n} t\right)+c c\right]\right\} \sin \omega_{n} x .
\end{aligned}
$$

Furthermore, the supplementary term of the natural frequency from a fractional derivative is

$$
\omega_{n a}=-\frac{N}{2 \omega_{n} \sigma_{n}} \sin \left(\sigma_{n} \varepsilon t\right) \int_{0}^{1} X_{n} X_{n}^{\prime \prime} d x+\frac{\eta}{2} \varepsilon t \omega_{n}^{\alpha-1} \cos \left(\frac{\pi}{2} \alpha\right) .
$$

The variation of a supplementary term from a fractional derivative according to $\alpha$-order is shown in Figure 2. The effects of the order of a fractional derivative on the displacementtime curves are seen readily in Figure 3. The damping accelerates acutely in the classic damping approach, namely $\alpha=1$.

\subsection{3 $\Omega_{1}$ close to $2 \omega_{n}$ and $\Omega_{2}$ away from $\omega_{n}$}

In this part, we get the principal parametric resonance such that $\Omega_{1}=2 \omega_{n}+\varepsilon \sigma_{n}$. Then the stability boundaries are

$$
\sigma_{n}=\eta \omega_{n}^{\alpha-1} \cos \left(\frac{\pi}{2} \alpha\right) \pm \sqrt{\frac{N^{2}}{4 \omega_{n}^{2}}\left(\int_{0}^{1} X_{n} X_{n}^{\prime \prime} d x\right)^{2}-\left(\eta \omega_{n}^{\alpha-1} \sin \left(\frac{\pi}{2} \alpha\right)\right)^{2}}
$$

Figure 4 shows the effects of $\alpha$-order on the critical value $N$ and the variation of an unstable region with some different values of $\alpha$. It is observed that the critical value $N$ becomes zero for $\alpha=0$. The unstable region diminishes as $\alpha$ increases.

\subsection{The dynamic analysis of an axially loaded viscoelastic beam resting on foundation}

The fractional viscoelastic beam with axial load is resting on linear elastic foundation. This type of foundation is known as Winkler foundation. In the linear Winkler foundation model, $\hat{k}$ denotes the soil coefficient. The beam is modelled by fractional Kelvin-Voight 


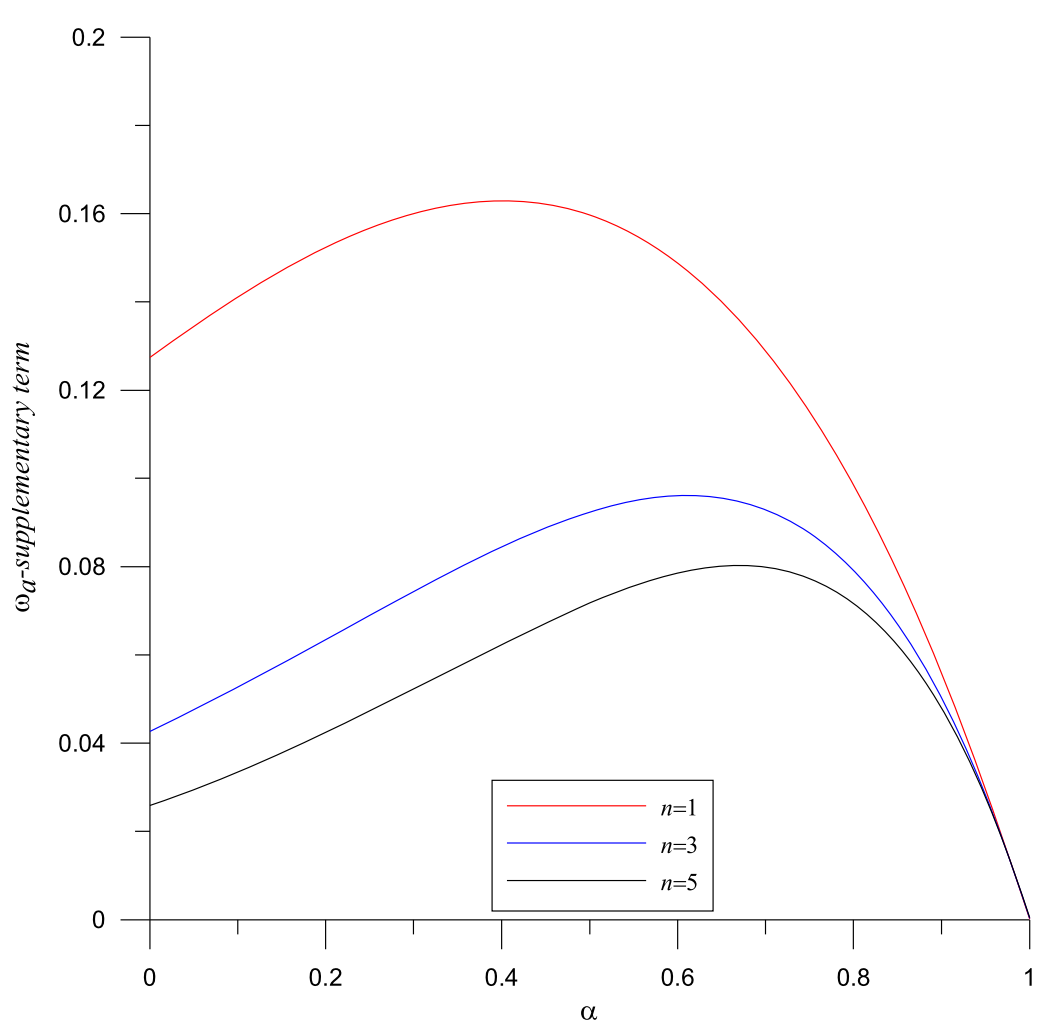

Figure 2 Supplementary term versus $\alpha$ for various mode values at $t=1(\varepsilon=0.1, \eta=8, N=1)$.

viscoelastic material. The governing equation is given by

$$
E I \frac{\partial^{4} \hat{w}}{\partial \hat{x}^{4}}+\hat{\eta} \frac{\partial^{\alpha}}{\partial \hat{t}^{\alpha}}\left(\frac{\partial^{4} \hat{w}}{\partial \hat{x}^{4}}\right)+P_{0} \frac{\partial^{2} \hat{w}}{\partial \hat{x}^{2}}+\hat{k} \hat{w}+m \frac{\partial^{2} \hat{w}}{\partial \hat{t}^{2}}=\hat{f}(\hat{x}) \cos \hat{\Omega} \hat{t}
$$

where $E$ represents the modulus of elasticity, $I$ is the moment of inertia and $P_{0}$ denotes axial force. Now, let us introduce the dimensionless quantities

$$
w=\frac{\hat{w}}{L}, \quad x=\frac{\hat{x}}{L}, \quad t=\frac{\hat{t}}{L} \sqrt{\frac{P_{0}}{m}}
$$

and the new dimensionless parameters are

$$
\begin{aligned}
& v_{f}^{2}=\frac{E I}{P_{0} L^{2}}, \quad \bar{\eta}=\frac{\hat{\eta}}{L^{\alpha+2}} \sqrt{\frac{P_{0}^{\alpha-2}}{m^{\alpha}}} \quad(\bar{\eta}=\varepsilon \eta), \\
& k=\frac{\hat{k} L^{2}}{P_{0}}, \quad f=\frac{\hat{f} L}{P_{0}}, \quad \Omega=\hat{\Omega} L \sqrt{\frac{m}{P_{0}}},
\end{aligned}
$$

where $v_{f}$ is the flexural stiffness coefficient. Then the dimensionless equation and the boundary conditions are obtained as

$$
\begin{aligned}
& v_{f}^{2} \frac{\partial^{4} w}{\partial x^{4}}+\varepsilon \eta \frac{\partial^{\alpha}}{\partial t^{\alpha}}\left(\frac{\partial^{4} w}{\partial x^{4}}\right)+\frac{\partial^{2} w}{\partial x^{2}}+k w+\frac{\partial^{2} w}{\partial t^{2}}=f(x) \cos \Omega t, \\
& w(0, t)=w(1, t)=0, \quad w^{\prime \prime}(0, t)=w^{\prime \prime}(1, t)=0 .
\end{aligned}
$$




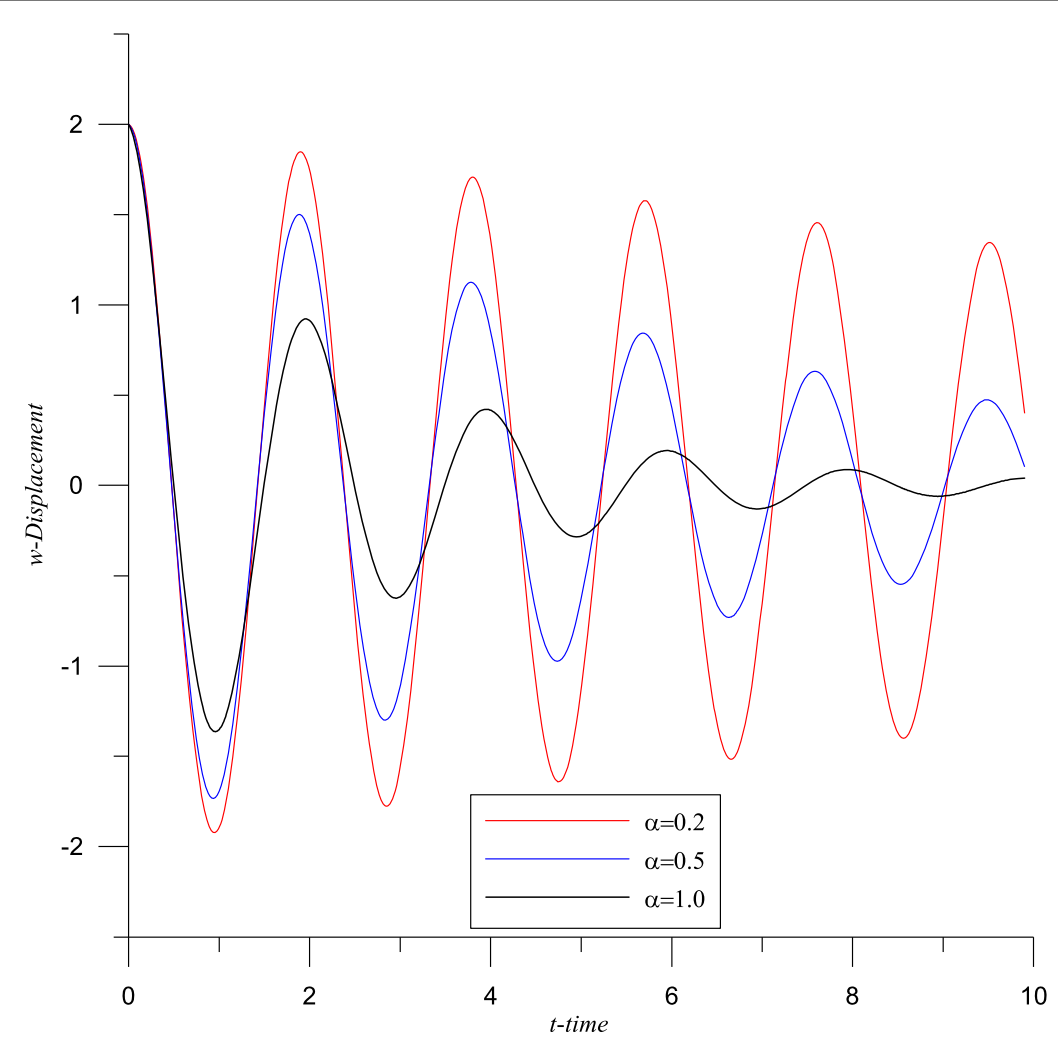

Figure 3 Displacement-time curves for the different values of $\alpha(\varepsilon=0.1, n=1, \eta=8.0, a=0.2$, $\sigma=0.1$ ).

Thus, the operators corresponding to a general model are

$$
\begin{aligned}
& L_{0}[w]=v_{f}^{2} w^{\prime \prime \prime \prime}+w^{\prime \prime}+k w, \\
& L_{1}[w]=0, \\
& L_{2}[w]=\eta D^{\alpha} w^{\prime \prime \prime \prime}
\end{aligned}
$$

and the other terms are

$$
\begin{aligned}
& F(x)=f(x), \\
& \Omega_{2}=\Omega .
\end{aligned}
$$

Then Eqs. (13) and (15) reduce to

$$
\begin{aligned}
& v_{f}^{2} X_{n}^{\prime \prime \prime \prime}+X_{n}^{\prime \prime}+\left(k-\omega_{n}^{2}\right) X_{n}=0, \\
& X_{n}(0)=X_{n}(1)=0, \quad X_{n}^{\prime \prime}(0)=X_{n}^{\prime \prime}(1)=0
\end{aligned}
$$

and

$$
\begin{aligned}
& v_{f}^{2} Y^{\prime \prime \prime \prime}+Y^{\prime \prime}+\left(k-\Omega^{2}\right) Y=\frac{1}{2} f(x), \\
& Y(0)=Y(1)=0, \quad Y^{\prime \prime}(0)=Y^{\prime \prime}(1)=0 .
\end{aligned}
$$




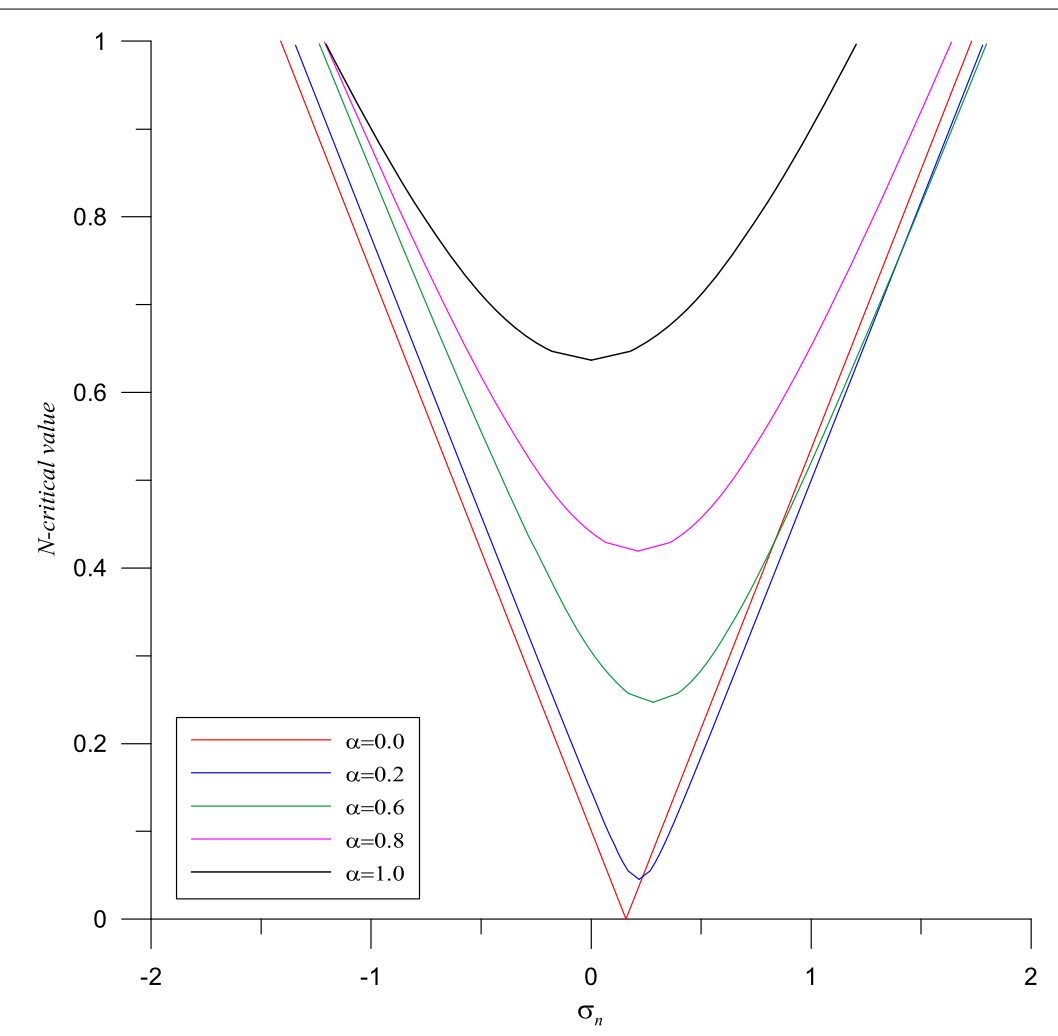

Figure 4 Stability boundaries for various fractional order for second mode $(\eta=1.0)$.

Finally, the solutions of Eqs. (82) and (84) are

$$
\begin{aligned}
X_{n}(x)= & c_{1 n}\left[e^{r_{1 n} x}+\frac{r_{4 n}^{2}-r_{1 n}^{2}}{r_{2 n}^{2}-r_{4 n}^{2}} \frac{e^{r_{3 n} x}-e^{r_{1 n} x}}{e^{r_{3 n} x}-e^{r_{2 n} x}} e^{r_{2 n} x}+\frac{r_{4 n}^{2}-r_{1 n}^{2}}{r_{3 n}^{2}-r_{4 n}^{2}} \frac{e^{r_{1 n} x}-e^{r_{2 n} x}}{e^{r_{3 n} x}-e^{r_{2 n} x}} e^{r_{3 n} x}\right. \\
& \left.-\left(1+\frac{r_{4 n}^{2}-r_{1 n}^{2}}{r_{2 n}^{2}-r_{4 n}^{2}} \frac{e^{r_{3 n} x}-e^{r_{1 n} x}}{e^{r_{3 n} x}-e^{r_{2 n} x}}+\frac{r_{4 n}^{2}-r_{1 n}^{2}}{r_{3 n}^{2}-r_{4 n}^{2}} \frac{e^{r_{1 n} x}-e^{r_{2 n} x}}{e^{r_{3 n} x}-e^{r_{2 n} x}}\right) e^{r_{4 n} x}\right]
\end{aligned}
$$

and

$$
Y(x)=\alpha_{1 n} e^{\beta_{1 n} x}+\alpha_{2 n} e^{\beta_{2 n} x}+\alpha_{3 n} e^{\beta_{3 n} x}+\alpha_{4 n} e^{\beta_{4 n} x}+\chi(x),
$$

where $\chi(x)$ represents the particular solution from a non-homogeneous part. For the solution at $\varepsilon$-order, two different cases arise as follows.

5.2.1 $\Omega_{1}$ away from $2 \omega_{n}$ and $0, \Omega_{2}$ away from $\omega_{n}$ Using the general solution (23), we get

$$
d_{1}=\eta \int_{0}^{1} X_{n}(x) X_{n}^{\prime \prime \prime \prime}(x) d x
$$

The amplitude and displacement are obtained

$$
A_{n}\left(T_{1}\right)=A_{0} \exp \left\{\frac{\eta}{2} \omega_{n}^{\alpha-1} T_{1}\left[i \cos \left(\frac{\pi}{2} \alpha\right)-\sin \left(\frac{\pi}{2} \alpha\right)\right] \int_{0}^{1} X_{n}(x) X_{n}^{\prime \prime \prime \prime}(x)(x) d x\right\}
$$


and

$$
\begin{aligned}
& w(x, t) \\
& \cong A_{0} \exp \left(-\frac{\eta}{2} \omega_{n}^{\alpha-1} \varepsilon t \sin \left(\frac{\pi}{2} \alpha\right) \int_{0}^{1} X_{n}(x) X_{n}^{\prime \prime \prime \prime}(x)(x) d x\right) \\
& \quad \times\left\{\exp \left[i\left(\frac{\eta}{2} \varepsilon \omega_{n}^{\alpha-1} \cos \left(\frac{\pi}{2} \alpha\right) \int_{0}^{1} X_{n}(x) X_{n}^{\prime \prime \prime \prime}(x)(x) d x+\omega_{n}\right) t\right]+c c\right\} X_{n}(x) .
\end{aligned}
$$

Thus, the supplementary term of the natural frequency due to a fractional derivative is

$$
\omega_{n a}=\frac{\eta}{2} \varepsilon \omega_{n}^{\alpha-1} \cos \left(\frac{\pi}{2} \alpha\right) \int_{0}^{1} X_{n}(x) X_{n}^{\prime \prime \prime \prime}(x)(x) d x .
$$

\subsection{2 $\Omega_{1}$ away from 0 and $2 \omega_{n}, \Omega_{2}$ close to $\omega_{n}$}

In this case, using Eq. (45), the stability boundaries which correspond to the primary resonance $\Omega_{2}=\omega_{n}+\varepsilon \sigma_{n}$ are given as

$$
\begin{aligned}
\sigma_{n}= & \frac{1}{2} \omega_{n}^{\alpha-1} \eta \cos \left(\frac{\pi}{2} \alpha\right) \int_{0}^{1} X_{n} X_{n}^{\prime \prime \prime \prime}(x) d x \\
& \pm \omega_{n}^{\alpha-1} \eta \sqrt{\frac{1}{a_{n}^{2}}\left(\int_{0}^{1} X_{n} Y^{\prime \prime \prime \prime}(x) d x\right)^{2}-\frac{1}{4} \sin ^{2}\left(\frac{\pi}{2} \alpha\right)\left(\int_{0}^{1} X_{n} X_{n}^{\prime \prime \prime \prime}(x) d x\right)^{2}}
\end{aligned}
$$

where the coefficient is

$$
d_{3}=\eta \int_{0}^{1} X_{n}(x) Y^{\prime \prime \prime \prime}(x) d x
$$

\section{Conclusion and discussions}

In this study, the general model subject to internal and external excitation is developed. The general model proposed for continuum is linear and one-dimensional. The effect of the damping term which is obtained from viscoelastic material properties is modelled with a fractional derivative. The dynamic analysis of the general model is examined by the method of multiple time scales. The approximate solutions are derived in terms of operators. The external force term is considered at order one. This consideration leads to sum and difference type of resonance in addition to primary and parametric resonance cases. The application of the general solution to two specific engineering problems is presented. The solvability boundaries are approximately obtained and numerically illustrated. It is shown that the order of the fractional derivative has an effect on natural frequencies and stability boundaries. It is shown that the stable region becomes smaller with increasing fractional order. And also, the coefficient of a fractional damping term has similar effects to fractional order. 


\section{Author details}

'Department of Mathematics, Faculty of Art \& Sciences, Celal Bayar University, Manisa, 45047, Turkey. ${ }^{2}$ Department of Civil Engineering, Faculty of Engineering, Celal Bayar University, Manisa, 45140, Turkey.

\section{Acknowledgements}

Dedicated to Professor Hari M Srivastava.

Received: 18 December 2012 Accepted: 12 April 2013 Published: 25 April 2013

\section{References}

1. Rossikhin, YA, Shitikova, MV: Application of fractional calculus for dynamic approximate analytical solutions problems of solid mechanics: novel trends and recent results. Appl. Mech. Rev. 63, 010801 (2010)

2. Debnath, L: Recent applications of fractional calculus to science and engineering. Int. J. Math. Math. Sci. $\mathbf{5 4}$ 3413-3442 (2003)

3. Pakdemirli, M: Vibrations of continuous systems with a general operator notation suitable for perturbative calculations. J. Sound Vib. 246(5), 841-851 (2001)

4. Özhan, B, Pakdemirli, M: A general solution procedure for the forced vibrations of a continuous system with cubic nonlinearities: primary resonance case. J. Sound Vib. 325(4-5), 894-906 (2009)

5. Özhan, B, Pakdemirli, M: A general solution procedure for the forced vibrations of a continuous system with cubic nonlinearities: three-to-one internal resonances with external excitation. J. Sound Vib. 329(13), 2603-2615 (2010)

6. Özhan, B, Pakdemirli, M: Principal parametric resonances of a general continuous system with cubic nonlinearities. Appl. Math. Comput. 219(5), 2412-2423 (2012)

7. Ghayesh, MH, Kazemirad, S, Darabi, MA: A general solution procedure for vibrations of systems with cubic nonlinearities and nonlinear/time-dependent internal boundary conditions. J. Sound Vib. 330(22), 5382-5400 (2011)

8. Agnieszka, B, Malinowskaa, D, Torres, FM: Generalized natural boundary conditions for fractional variational problems in terms of the Caputo derivative. Comput. Math. Appl. 59, 3110-3116 (2010)

9. Kilbas, AA, Srivastava, HM, Trujillo, JJ: Theory and Applications of Fractional Differential Equations. North-Holland Mathematics Studies, vol. 204. Elsevier, Amsterdam (2006)

10. Deü, JF, Matignon, D: Simulation of fractionally damped mechanical systems by means of a Newmark-diffusive scheme. Comput. Math. Appl. 59, 1745-1753 (2010)

11. Bagley, RL, Calico, RA: The fractional order state equations for the control of viscoelastically damped structures. J. Guid. Control Dyn. 14(2), 304-311 (1991)

12. Bagley, RL, Torvik, PJ: Fractional calculus - a different approach to the analysis of viscoelastically damped structures. AIAA J. 21(5), 741-748 (1983)

13. Bagley, RL, Torvik, PJ: Fractional calculus in the transient analysis of viscoelastically damped structures. AIAA J. 23(6), 918-925 (1985)

14. Rossikhin, YA, Shitikova, MV: A new method for solving dynamic problems of fractional derivative viscoelasticity. Int J. Eng. Sci. 39, 149-176 (2001)

15. Mainardi, F: Fractional calculus: some basic problems in continuum and statistical mechanics. In: Carpinteri, A, Mainardi, F (eds.) Fractals and Fractional Calculus in Continuum Mechanics. CISM Courses and Lectures, vol. 378, pp. 291-348. Springer, New York (1997)

16. Cooke, JA, Keltie, RF: Determination of the impulse response of a viscoelastic beam using a fractional derivative constitutive model. ASME Des. Engineering Division Publications. DE 5, 137-141 (1987)

17. Skaar, SB, Michel, AN, Miller, RK: Stability of viscoelastic control systems. IEEE Trans. Autom. Control 33(4), 348-357 (1988)

18. French, M, Rogers, J: A survey of fractional calculus for structural dynamics applications. In: IMAC-IX: A Conference on Structural Dynamics, Kissimmee, FL, pp. 305-309 (2001)

19. Demir, DD, Bildik, N, Sinir, BG: Application of fractional calculus in the dynamics of beams. Bound. Value Probl. 135 1-13 (2012)

20. Demir, DD, Bildik, N, Sinir, BG: Linear dynamical analysis of fractionally damped beams and rods. J. Eng. Math. (2013). doi:10.1007/s10665-013-9642-9

21. Pakdemirli, $\mathrm{M}$, Boyacı, $\mathrm{H}$ : The direct-perturbation methods versus the discretization-perturbation method: linear systems. J. Sound Vib. 199(5), 825-832 (1997)

22. Rossikhin, YA, Shitikova, MV: On fallacies in the decision between the Caputo and Riemann-Liouville fractional derivatives for the analysis of the dynamic response of a nonlinear viscoelastic oscillator. Mech. Res. Commun. 45 22-27 (2012)

23. Nayfeh, AH: Introduction to Perturbation Techniques. Wiley, New York (1981)

24. Chen, LQ, Zhao, WJ, Zu, JW: Transient responses of an axially accelerating viscoelastic string constituted by a fractional differentiation law. J. Sound Vib. 278, 861-871 (2004)

doi:10.1186/1687-2770-2013-104

Cite this article as: Dönmez Demir et al.: Linear vibrations of continuum with fractional derivatives. Boundary Value Problems 2013 2013:104 